\title{
Case of the Irretrievable Inferior Vena Cava Filter
}

\author{
Arjun Jayaraj $^{1 *}$, Joe Ference ${ }^{2}$, Sanjiv Parikh² and Kaj Johansen² \\ 'University of Washington, Seattle, WA, USA
}

${ }^{2}$ Swedish Medical Center, Seattle, WA, USA

\section{Case Report}

With increasing use of Inferior Vena Cava (IVC) filters, more reports are emerging of complications includingdislocation of the filter $[1,2]$.We report the case of a 16 year old lady, who developed extensive right ileo-femoral deep vein thrombosis (with extension into Inferior Vena Cava [IVC]) and pulmonary embolismin a setting of oral contraceptive use. During her work up following presentation she was noted to be heterozygous for Factor $\mathrm{V}$ Leiden. Following diagnosis she underwent successful pharmaco-mechanical thrombectomy and balloon angioplasty of the involvedileo-femoral venous segment and IVC with consequent resolution of pain and swelling of her right leg. At that same time she also underwent placement of a prophylactic IVC filter (suprarenal Bard G2 filter [Bard Peripheral Vascular, Inc; Tempe, AZ]). Completion venogram demonstrated recanalization of the IVC and the right ileo-femoral segment. The filter was also demonstrated to be in good position. Subsequently, multiple attemptsat removal of this temporary filter three months post placement were unsuccessful. The approaches applied included tandem techniques via trans-jugular and trans-femoral approaches. This was likely due to the fact thatthe filter had taken a horizontal disposition (prior to retrieval attempts) just above the level of the renal veins with herniation of struts into the left renal vein. Such a malpositioned filter brought with it the risk of migration and possible cavalocclusion (7\%-10\% per decade) [3]. Additionally there was also the risk of renal vein thrombosis secondary to herniation of the struts into the left renal vein. While the patient had initially been asymptomatic (for 6 months following thrombolysis),

she went on to developpersistent flank and back pain. In the setting of a symptomatic malpositioned IVC filter who had previously failed multiple percutaneous attempts at filter removal, it was decided to proceed with open retrieval of the filter (10 months post placement). This was done via a right subcostal incision with the patient in a left lateral decubitus position. After adequate exposure of the perirenal inferior vena cava, control both proximally and distally of the IVC was obtained. The renal veins were also isolated and controlled subsequent to which the IVC was entered via an oblique incision and the filter retrieved. Delivery of the filter required careful dissection as it involved peeling away endothelialized struts from the surface of the IVC and renal veins. Some of the struts had to be cut away with a wire cutter. Following delivery of the filter, the defect in the IVC was repaired using a bovine pericardial patch.

The patient recovered quickly from this operation and at this time, 2 months post procedure she is back to her normal routine with complete resolution of symptoms.

\section{References}

1. Iliescu B, Haskal ZJ (2012) Advanced Techniques for Removal of Retrievable Inferior Vena Cava Filters.Cardiovasc Intervent Radiol. 35: 741-750.

2. Binkert CA, Drooz AT, Caridi JG (2009) Technical success and safety of retrieval of the $\mathrm{G} 2$ filter in a prospective, multicenter study. J VasclntervRadiol20:1449-1453.

3. Proctor MC, Greenfield LJ (2004) Thirty-year Greenfield filter experience: are temporary filters needed? Vascular 12:S18.
*Corresponding author: Arjun Jayaraj, University of Washington, Box 356410 Seattle, WA 98195, USA, Tel: 2068836090; E-mail: jarjun@gmail.com

Received February 16, 2014; Accepted August 20, 2014; Published August 22 , 2014

Citation: Jayaraj A, Ference J, Parikh S, Johansen K (2014) Case of the Irretrievable Inferior Vena Cava Filter. J Vasc Med Surg 2: 151. doi: 10.4172/23296925.1000151

Copyright: @ 2014 Jayaraj A, et al. This is an open-access article distributed under the terms of the Creative Commons Attribution License, which permits unrestricted use, distribution, and reproduction in any medium, provided the original author and source are credited. 